\title{
Verändert die nCPAP-Therapie die Lungenfunktion bei Patienten mit obstruktivem Schlafapnoe-Syndrom?
}

\author{
W. Klinnert ${ }^{1}$ \\ M. Orth ${ }^{1}$ \\ K. Rasche ${ }^{2}$ \\ T. T. Bauer ${ }^{1}$ \\ G. Schultze-Werninghaus ${ }^{1}$ \\ H.-W. Duchna'
}

\author{
Does nCPAP-Therapy Change the Lung Function of Patients with Obstructive \\ Sleep Apnea Syndrome?
}

\section{Zusammenfassung}

Hintergrund: Die nCPAP-Therapie ist Standard bei Patienten mit obstruktivem Schlafapnoesyndrom (OSAS). Ziel der Studie war es herauszufinden, ob die Therapie innerhalb von drei Jahren die Lungenfunktion der Patienten verändert. Patienten und Methode: Untersucht wurde die Lungenfunktion von 228 Patienten mit einem OSAS ohne koprävalente Lungenerkrankung, die erstmalig auf eine nCPAP-Therapie eingestellt wurden mittels Ganzkörperplethysmographie, Spirometrie und Blutgasanalyse. Nach 1, 2 und 3 Jahren wurde die Lungenfunktion der kontrolluntersuchten Patienten mit den individuellen Ergebnissen der Ausgangsmessung verglichen. Ergebnisse: In der Ausgangs- sowie in den Folgeuntersuchungen wiesen alle Patienten eine durchschnittlich normale Lungenfunktion auf. Bei allen Nachuntersuchungen wurde insbesondere bei den Rauchern eine signifikante Verbesserung der inspiratorischen Vitalkapazität (\%Soll) gemessen. Bei den dynamischen Parametern wurde eine signifikante Abnahme der maximalen exspiratorischen Atemstromstärke bei 50\% der Vitalkapazität (MEF 50\%Soll) nach 1 und 2 Jahren und des Tiffeneau-Wertes $\left(\mathrm{FEV}_{1} \% \mathrm{VC}\right)$ nach 2 und 3 Jahren bei unveränderter Einsekundenkapazität $\left(\mathrm{FEV}_{1}\right)$ registriert. Schlussfolgerungen: Obstruktive oder restriktive Ventilationsstörungen wurden unter nCPAP-Therapie im Beobachtungsintervall von bis zu drei Jahren nicht beobachtet. In der vorliegenden retrospektiven Untersuchung wurde unter nCPAP-Therapie eine Zunahme der Vi-

\section{Abstract}

Background: nCPAP-therapy is standard for patients with obstructive sleep apnea syndrome (OSAS). This study investigated, if nCPAP changed the patients lung function. Patients and Methods: Lung function of 228 OSAS patients without coprevalent lung disease, who received nCPAP for the first time, was examined (whole bodyplethysmography, spirometry, and blood gas analysis). After 1, 2, and 3 years the results of the re-examined patients were compared with their individual starting results. Results: The results of the patients in the basic and in the follow-up examinations was within standard range. During reexamination, an improvement of inspiratory vital capacity was registered repetitively, especially in smokers. On the other hand, MEF 50 decreased within the first two years and $\mathrm{FEV}_{1} \% \mathrm{VC}$, but not $\mathrm{FEV}_{1}$, decreased after 2 and 3 years of reexamination. Conclusions: Neither obstructive nor restrictive lung diseases were diagnosed under nCPAP-therapy within three years. This study with a retrospective design showed significant improvements of vital capacity (VC) in patients with OSAS, especially in smokers, under nCPAP-therapy. This increase can be explained by an effect of practice in the examination and by recruitment of formerly hypoventilated lung areas. As MEF 50 and $\mathrm{FEV}_{1} \% \mathrm{VC}$ depend on VC, the decrease of both parameters may be caused by the increase of VC. In summary, nCPAP did not cause negative effects of lung function in the investigated patients over a period of 3 years.

${ }^{1}$ BG-Kliniken Bergmannsheil, Universitätsklinik, Klinikum der Ruhr-Universität Bochum; Medizinische Klinik III, Pneumologie, Allergologie, Schlaf- und Beatmungsmedizin;

(Univ.-Prof. Dr. med. G. Schultze-Werninghaus)

${ }^{2}$ Kliniken St. Antonius, Akademisches Lehrkrankenhaus für die Universität Düsseldorf, Zentrum für Innere Medizin, 2. Medizinische Klinik, Schwerpunkt Pneumologie; (Prof. Dr. med. K. Rasche)

Korrespondenzadresse

Priv.-Doz. Dr. Hans-Werner Duchna · BG-Kliniken Bergmannsheil, Universitätsklinik · Klinikum der Ruhr-Universität Bochum · Medizinische Klinik III · Pneumologie, Allergologie, Schlaf- und Beatmungsmedizin · Bürkle-de-la-Camp-Platz 1 . 44789 Bochum

E-mail: hans-werner.duchna@ruhr-uni-bochum.de

Eingang: 16. Mai 2004 · Nach Revision alkzeptiert: 25. Juni 2004

Bibliografie

Pneumologie 2004; 58: 712-717 @ Georg Thieme Verlag KG Stuttgart · New York DOI $10.1055 / \mathrm{s}-2004-830042$

ISSN 0934-8387 
talkapazität gezeigt, die am ehesten durch einen Übungseffekt bei der Durchführung des Untersuchungsmanövers und durch Rekrutierung minderbelüfteter Lungenareale erklärbar ist. Verschlechterungen der MEF 50- sowie der Tiffeneau-Werte bei unveränderter Einsekundenkapazität sind durch die Relation zur Verbesserung der Vitalkapazität erklärbar. Somit konnte ein negativer Einfluss der nCPAP-Therapie auf die Lungenfunktion im Patientenkollektiv im Verlauf von 3 Jahren nicht gezeigt werden.

\section{Einleitung}

Die nasale Überdruck (nCPAP)-Therapie gilt heutzutage als Standardtherapie des obstruktiven Schlafapnoe-Syndroms (OSAS) und wurde erstmals 1981 von Sullivan u. Mitarb. erfolgreich angewendet [1 - 3]. Da diese etablierte Therapieform durch den Patienten über viele Jahre durchgeführt werden muss, sind neben der nachgewiesenen Effektivität bezüglich der Schlafapnoetherapie [4] Auswirkungen auf die Atemmechanik, bedingt durch die Applikation des positiven Überdruckes denkbar $[5,6]$. Bisher veröffentlichte Untersuchungen zu einer möglichen Beeinträchtigung der Lungenfunktion durch eine langjährige nCPAP-Applikation lassen keine eindeutigen Aussagen zu. So haben Hein und Magnussen in ihrer Studie Patienten mit einem OSAS nach $27 \pm$ 23 Monaten nCPAP-Therapie untersucht und dabei keine relevanten Veränderungen der Lungenfunktionsparameter festgestellt [7]. Dahingegen zeigten Chaouat u. Mitarb. in ihrem Patientenkollektiv eine signifikante Verschlechterung der Einsekundenkapazität $\left(\mathrm{FEV}_{1}\right)$ nach einer nCPAP-Therapiezeit von fünf Jahren (64 \pm 6 Monate) [8]. In verschiedenen Studien konnte gezeigt werden, dass der Sauerstoffpartialdruck vor allem bei hypoxämischen Patienten, aber auch der Kohlendioxidpartialdruck bei Personen mit Hyperkapnie, durch die nCPAP-Therapie positiv beeinflussbar ist [8-10]. Eine prospektive, randomisierte, doppelblinde Studie zur Auswirkung der nCPAP-Therapie auf die Lungenfunktion über mehrere Jahre ist aufgrund der gesicherten therapeutischen Effekte dieser als Goldstandard für das OSAS angesehenen Therapieform ethisch nicht vertretbar und technisch schwer durchführbar. Daher wurde in der vorliegenden retrospektiven Studie untersucht, ob es im Verlauf von bis zu drei Jahren bei Patienten mit OSAS unter nCPAP-Therapie zu Veränderungen der Lungenfunktion und der Blutgaswerte kommt.

\section{Material und Methoden}

Für die hier vorliegende retrospektive Studie wurden aus den Patientenakten des Schlaflabors der Berufsgenossenschaftlichen Kliniken Bergmannsheil, Universitätsklinik der Ruhr-Universität Bochum, diejenigen herausgesucht, bei denen beim Patienten ein Schlafapnoesyndrom mittels Polysomnographie diagnostiziert wurde, eine Ersteinstellung auf nCPAP erfolgte und eine Lungenfunktionsprüfung durchgeführt wurde (Zeitraum: 1992 - 1999). Ausgeschlossen wurden Patienten mit koprävalenter Lungenerkrankung (Anamnese, Arztberichte, Lungenfunktion). Weitere Erkrankungen, wie beispielsweise eine arterielle Hypertonie, waren jedoch kein Ausschlusskriterium. Als weitere Vorraussetzung musste jeder Patient im Verlauf seiner Therapie zu mindestens einer Kontrolluntersuchung im Schlaflabor erschienen sein. Entscheidende Kriterien für die Aufnahme eines Patienten in die Studie aber waren die durchgeführten Lungenfunktionsprüfungen sowohl vor der Ersteinstellung auf nCPAPTherapie als auch bei den Kontrolluntersuchungen, sowie eine regelmäßige Anwendung der nCPAP-Therapie während des Schlafes. Die nCPAP-Compliance wurde anamnestisch und durch Kontrolle der Geräte-Nutzungszeit überprüft [11]. Nichtkompliante Patienten oder Therapieabbrecher wurden von der Studienteilnahme ausgeschlossen. Das Rauchverhalten wurde ebenfalls erfasst.

Die Lungenfunktionsprüfungen wurden bei allen Patienten vor Einstellung auf die nCPAP-Therapie und im Verlauf bei jeder Kontrolluntersuchung tagsüber durchgeführt. Sie erfolgten mit den Geräten Body-Test ${ }^{\circledR}$ und Masterlab ${ }^{\circledR}$ der Fa. Jäger, Würzburg sowie mit dem $995^{\circledR}$ und Omni ${ }^{\circledR}$ Modular System der Fa. AVL/Roche. Die in der Untersuchung angewandten Normwerte wurden von der Expertenkommission der Europäischen Gemeinschaft für Kohle und Stahl (EGKS) [12] ermittelt. Durchgeführt wurden: Spirometrie einschließlich Fluss-Volumen-Diagramm, Ganzkörperplethysmographie und eine kapilläre Blutgasanalyse. Aus den vorhandenen Parametern wurden rechnerische Werte, wie die Totale Lungenkapazität (TLC) oder das Residualvolumen (RV) ermittelt. Je nach Aussagekraft wurden im Folgenden die Absolutwerte und/oder Relativwerte im Vergleich zu einer „Sollwert"-gesunden Population gleichen Alters, Geschlechts, Gewichts und gleicher Größe angegeben [12]. Allen Patienten wurde eine mindestens jährliche Kontrolle im Schlaflabor entsprechend den Empfehlungen der Deutschen Gesellschaft für Schlafforschung und Schlafmedizin (DGSM) und der Deutschen Gesellschaft für Pneumologie (DGP) empfohlen [3]. Aus unterschiedlichen nicht näher erfassten Gründen erschienen jedoch nicht alle Patienten regelmäßig zu den vorgegebenen Kontrolluntersuchungen. Daher erfolgte bei allen Kontrolluntersuchungen ein Vergleich mit den individuellen Ausgangswerten der an der jeweiligen Kontrolluntersuchung teilnehmenden Patienten nach 1,2 und 3 Jahren.

Die statistische Auswertung erfolgte mit dem Student's t-Test für verbundene Stichproben bei Normalverteilung beziehungsweise mit dem Wilcoxon-Signed-Rank-Test bei fehlender Normalverteilung. Das Signifikanzniveau wurde auf $5 \%$ festgelegt $(p<0,05)$. Angegeben wurden jeweils die Mittelwerte \pm einfache Standardabweichung.

\section{Ergebnisse}

228 Patienten mit einem OSAS (mittleres Alter 56,17 \pm 9,47 Jahre; 36 Frauen und 192 Männer) erfüllten die Ein- und Ausschlusskriterien und konnten somit in die Untersuchung eingeschlossen werden. Darunter befanden sich 152 (66,7\%) Nichtraucher, 69 (30,3\%) aktive Raucher und 7 (3\%) Personen ohne Angaben zum Rauchverhalten. Der nCPAP-Therapiedruck wurde auf durchschnittlich $9,83 \pm 2,33 \mathrm{~cm} \mathrm{H}_{2} \mathrm{O}$ eingestellt. Hierunter wurde polysomnographisch dokumentiert bei allen Patienten eine weitgehende Normalisierung des Schlafprofils mit Unterdrückung der Weckreaktionen unter Senkung des Apnoe-Hypopnoe-Index (AHI) von initial $41,6 \pm 25,11$ auf $7,82 \pm 15,11 /$ Stunde erreicht. 
Von diesen 228 Patienten erschienen zur Nachuntersuchung 142 nach 1 Jahr, 91 nach 2 und 67 nach 3 Jahren. Bei den im Folgenden dargestellten Messwerten ist zu beachten, dass der Vergleich der Verlaufsparameter jeweils mit den individuellen Ausgangswerten der an der jeweiligen Kontrolluntersuchung teilnehmenden Patienten erfolgte. Die Kollektive bei den Nachuntersuchungen waren hinsichtlich der anthropometrischen Daten (Altersund Geschlechtsverteilung, Body-Mass-Index) nicht unterschiedlich und zeigten, abgesehen vom Alter, keine signifikanten Veränderungen zum Gesamtkollektiv bei der Ausgangsmessung. Es wurden keine signifikante Veränderungen des Body-Mass-Index (BMI) im Beobachtungsintervall von $1 \mathrm{Jahr}$ (BMI-Ausgangswert $30,55 \pm 5,03$ vs. $30,60 \pm 4,90$ nach $1 \mathrm{Jahr}$, nicht signifikant [n.s.]), nach 2 Jahren (BMI-Ausgangswert $31,67 \pm 6,01$ vs. $31,85 \pm 6,44$ nach 2 Jahren, n. s.) bzw. nach 3 Jahren (BMI-Ausgangswert $31,22 \pm 5,15$ vs. $31,45 \pm 5,02$ nach 3 Jahren, n. s.) beobachtet.

Die 142 nach 1 Jahr nachuntersuchten Patienten, darunter 23 Frauen, wendeten ihre nCPAP-Therapie durchschnittlich 5,35 Stunden/Nacht an. Der nCPAP-Druck wurde im Rahmen der Kontrolluntersuchung nicht signifikant von 9,23 $\pm 1,96$ auf 9,48 \pm $2,9 \mathrm{~cm} \mathrm{H}_{2} \mathrm{O}$ angehoben. Hierunter wurde bei allen Patienten eine zufriedenstellende Therapie des OSAS dokumentiert. Während sich im Beobachtungsintervall keine signifikanten Veränderungen bei der Blutgasanalyse zeigten, wurde lungenfunktionsanalytisch eine signifikante Abnahme des MEF 50 (\%Soll) dokumentiert (Tab.1). Die in diesem Kollektiv enthaltenen 42 Raucher wiesen eine signifikante Verbesserung der inspiratorischen Vitalkapazität (VCIN, \%Soll) und konsekutiv eine Verringerung des Tiffeneau-Wertes $\left(\mathrm{FEV}_{1} \% \mathrm{VC}\right)$ bei unveränderter Einsekundenkapazität auf (Tab. 4).

Nach 2 Jahren wurden 91 Patienten (15 Frauen), die ihr nCPAPGerät durchschnittlich 5,2 Stunden/Nacht anwendeten, nachuntersucht. Der nCPAP-Druck musste bei der Nachuntersuchung von $9,84 \pm 2,14$ auf $10,64 \pm 2,21 \mathrm{~cm} \mathrm{H}_{2} \mathrm{O}$ angepasst werden $(p<0,05)$, um eine adäquate Therapie des OSAS zu gewährleisten. Es zeigten sich keine signifikanten Veränderungen der Blutgaswerte, jedoch eine Verbesserung der VCIN (\%Soll), der totalen Lungenkapazität (TLC), eine Zunahme des Residualvolumens in Prozent der TLC (RV\%TLC, \%Soll) sowie eine Verringerung des MEF 50 (\%Soll, $\mathrm{p}<0,05$ ) und von $\mathrm{FEV}_{1} \% \mathrm{VC}$, (absolut und \%Soll, $\mathrm{p}<0,05$ ) bei nicht signifikanter Verringerung des $\mathrm{FEV}_{1}$-Wertes (Tab. 2). Die Raucher in diesem Kollektiv ( $n=23)$ wiesen eine signifikante Abnahme des Tiffeneau-Wertes (absolut und \%Soll) auf (Tab. 4).

67 Patienten (8 Frauen) erschienen zur Nachuntersuchung nach 3 Jahren. Sie wendeten die CPAP-Therapie durchschnittlich 6,33 Stunden/Nacht an. Im 3. Therapiejahr wurde der nCPAP-Druck von $10,0 \pm 2,53$ auf $10,98 \pm 2,66 \mathrm{~cm} \mathrm{H}_{2} \mathrm{O}$ angehoben $(\mathrm{p}<0,05)$. In diesem Kollektiv zeigte sich im Verlauf eine signifikante Senkung des $\mathrm{PaCO}_{2}$, eine Verbesserung der VCIN (\%Soll), sowie bei den Rauchern eine Zunahme von VCIN (\%Soll) und konsekutiv eine Abnahme des Tiffeneau-Wertes (absolut und \%Soll; Tab. 3, Tab. 4, jeweils $\mathrm{p}<0,05$ ) bei unverändertem $\mathrm{FEV}_{1}$.

Zusammengefasst wiesen alle Patienten in der Ausgangs- sowie in den Folgeuntersuchungen eine durchschnittlich normale Lun-
Tab. 1 Verlauf der Lungenfunktionsparameter und der Blutgasanalyse bei allen nach 1 Jahr nCPAP-Therapie untersuchten Patienten ( $n=142$ Personen). n. s. = nicht signifikanter Unterschied bei einem Signifikanzniveau von $\mathrm{p}<0,05$

\begin{tabular}{|c|c|c|c|}
\hline & $\begin{array}{l}\text { Ausgangsmittel- } \\
\text { wert } \pm \text { Standard- } \\
\text { abweichung }\end{array}$ & $\begin{array}{l}\text { Mittelwert nach } \\
1 \text { Jahr nCPAP } \pm \\
\text { Standardabweichung }\end{array}$ & Signifikanz \\
\hline $\begin{array}{l}\mathrm{PaO}_{2}(\mathrm{~mm} \mathrm{Hg}) \\
\mathrm{PaCO}_{2}(\mathrm{~mm} \mathrm{Hg})\end{array}$ & $\begin{array}{l}79,60 \pm 9,53 \\
40,33 \pm 3,31\end{array}$ & $\begin{array}{l}80,42 \pm 9,87 \\
40,07 \pm 3,17\end{array}$ & $\begin{array}{l}\text { n.s. } \\
\text { n.s. }\end{array}$ \\
\hline Rt (hpa/l/s) & $3,04 \pm 1,46$ & $2,96 \pm 1,40$ & n.s. \\
\hline ITGV (\%Soll) & $93,84 \pm 22,22$ & $94,49 \pm 21,06$ & n.s. \\
\hline PEF (\%Soll) & $111,44 \pm 6,38$ & $113,10 \pm 24,62$ & n.s. \\
\hline MEF 50 (\%Soll) & $77,35 \pm 30,09$ & $74,54 \pm 30,70$ & $p<0,05$ \\
\hline VCIN (\%Soll) & $97,30 \pm 14,64$ & $98,06 \pm 15,13$ & n.s. \\
\hline$F E V_{1}(\%$ Soll) & $97,21 \pm 19,34$ & $96,72 \pm 9,06$ & n.s. \\
\hline$F E V_{1} \%$ VC (\%) & $76,76 \pm 9,36$ & $75,85 \pm 9,18$ & n.s. \\
\hline$F E V_{1} \%$ VC (\%Soll) & $99,54 \pm 11,86$ & $98,17 \pm 11,69$ & n.s. \\
\hline TLC (\%Soll) & $98,10 \pm 12,88$ & $97,58 \pm 12,76$ & n.s. \\
\hline RV\%TLC (\%Soll) & $99,70 \pm 19,23$ & $100,17 \pm 18,61$ & n.s. \\
\hline
\end{tabular}

Tab. 2 Verlauf der Lungenfunktionsparameter und der Blutgasanalyse bei allen nach 2 Jahren nCPAP-Therapie untersuchten Patienten ( $n=91$ Personen). n. s. = nicht signifikanter Unterschied bei einem Signifikanzniveau von $\mathrm{p}<0,05$

\begin{tabular}{|c|c|c|c|}
\hline & $\begin{array}{l}\text { Ausgangsmittel- } \\
\text { wert } \pm \text { Standard- } \\
\text { abweichung }\end{array}$ & $\begin{array}{l}\text { Mittelwert nach } \\
2 \text { Jahren nCPAP } \pm \\
\text { Standardabweichung }\end{array}$ & Signifikanz \\
\hline $\begin{array}{l}\mathrm{PaO}_{2}(\mathrm{~mm} \mathrm{Hg}) \\
\mathrm{PaCO}_{2}(\mathrm{~mm} \mathrm{Hg})\end{array}$ & $\begin{array}{l}78,51 \pm 9,75 \\
40,34 \pm 3,63\end{array}$ & $\begin{array}{l}77,18 \pm 10,14 \\
41,20 \pm 5,95\end{array}$ & $\begin{array}{l}\text { n.s. } \\
\text { n.s. }\end{array}$ \\
\hline Rt (hpa/l/s) & $3,03 \pm 1,38$ & $3,02 \pm 1,47$ & n.s. \\
\hline ITGV (\%Soll) & $95,31 \pm 21,57$ & $98,02 \pm 20,64$ & n.s. \\
\hline PEF (\%Soll) & $104,34 \pm 23,53$ & $103,82 \pm 24,03$ & n.s. \\
\hline MEF 50 (\%Soll) & $77,67 \pm 31,53$ & $69,67 \pm 30,63$ & $p<0,05$ \\
\hline VCIN (\% Soll) & $94,17 \pm 15,37$ & $95,03 \pm 18,06$ & $p<0,05$ \\
\hline FEV ${ }_{1}$ (\%Soll) & $94,92 \pm 0,39$ & $92,71 \pm 21,89$ & n.s. \\
\hline$F E V_{1} \% V C(\%)$ & $76,82 \pm 9,10$ & $74,58 \pm 9,60$ & $p<0,05$ \\
\hline FEV ${ }_{1} \% V C$ (\%Soll) & $100,15 \pm 10,84$ & $96,96 \pm 12,38$ & $p<0,05$ \\
\hline TLC (\%Soll) & $95,69 \pm 13,58$ & $98,20 \pm 11,73$ & $p<0,05$ \\
\hline RV\%TLC (\%Soll) & $101,01 \pm 2,14$ & $108,18 \pm 22,40$ & $p<0,05$ \\
\hline
\end{tabular}

genfunktion auf. Bei keinem Patienten wurde im Beobachtungsintervall eine obstruktive oder restriktive Ventilationsstörung unter nCPAP-Therapie diagnostiziert. In der vorliegenden retrospektiven Untersuchung wurde unter nCPAP-Therapie sowohl nach 1, 2 und 3 Jahren eine Verbesserung der VCIN, insbesondere bei den Rauchern gesehen. Verschlechterungen der MEF50-Werte (nach 1 und 2 Jahren) sowie der Tiffeneau-Werte (nach 2 und 3 Jahren) bei unverändertem $\mathrm{FEV}_{1}$ sind, da rechnerisch von der Vitalkapazität abhängig, in Relativität zur Verbesserung der Vitalkapazität zu bewerten. Veränderungen bei der TLC und RV\%TLC traten nur einmalig nach 2 Jahren auf und ließen sich nicht weiter verfolgen (Tab. 2). 
Tab. 3 Verlauf der Lungenfunktionsparameter und der Blutgasanalyse bei allen nach 3 Jahren untersuchten Patienten $(n=67$ Personen). n. s. = nicht signifikanter Unterschied bei einem Signifikanzniveau von $\mathrm{p}<0,05$

\begin{tabular}{|c|c|c|c|}
\hline & $\begin{array}{l}\text { Ausgangsmittel- } \\
\text { wert } \pm \text { Standard- } \\
\text { abweichung }\end{array}$ & $\begin{array}{l}\text { Mittelwert nach } \\
\text { 3 Jahren nCPAP } \pm \\
\text { Standardabweichung }\end{array}$ & Signifikanz \\
\hline $\begin{array}{l}\mathrm{PaO}_{2}(\mathrm{~mm} \mathrm{Hg}) \\
\mathrm{PaCO}_{2}(\mathrm{~mm} \mathrm{Hg})\end{array}$ & $\begin{array}{l}80,19 \pm 8,81 \\
40,41 \pm 3,52\end{array}$ & $\begin{array}{r}78,8 \pm 8,75 \\
39,43 \pm 2,86\end{array}$ & $\begin{array}{l}\text { n.s. } \\
p<0,05\end{array}$ \\
\hline Rt (hpa/l/s) & $3,08 \pm 1,54$ & $3,27 \pm 1,63$ & n.s. \\
\hline ITGV (\%Soll) & $89,06 \pm 3,32$ & $89,95 \pm 19,23$ & n.s. \\
\hline PEF (\%Soll) & $104,99 \pm 24,72$ & $108,40 \pm 30,23$ & n.s. \\
\hline MEF 50 (\%Soll) & $82,79 \pm 32,27$ & $77,11 \pm 32,61$ & n.s. \\
\hline VCIN (\%Soll) & $92,69 \pm 14,58$ & $95,10 \pm 15,75$ & $p<0,05$ \\
\hline FEV (\%Soll) & $95,11 \pm 19,30$ & $95,31 \pm 22,49$ & n.s. \\
\hline$F E V_{1} \% V C(\%)$ & $78,64 \pm 8,61$ & $77,07 \pm 10,32$ & n.s. \\
\hline FEV ${ }_{1} \%$ VC (\%Soll) & $101,53 \pm 10,53$ & $100,15 \pm 12,90$ & n.s. \\
\hline TLC (\% Soll) & $93,07 \pm 12,23$ & $94,81 \pm 9,64$ & n.s. \\
\hline RV\%TLC (\%Soll) & $98,75 \pm 24,89$ & $100,24 \pm 19,45$ & n.s. \\
\hline
\end{tabular}

Tab. 4 Verlauf der signifikant veränderten Lungenfunktionsparameter bei den Rauchern nach 1, 2 bzw. 3 Jahren nCPAPTherapie bei einem Signifikanzniveau von $p<0,05$

\begin{tabular}{lcll}
\hline & $\begin{array}{l}\text { Ausgangsmittel- } \\
\text { wert } \pm \text { Standard- } \\
\text { abweichung }\end{array}$ & $\begin{array}{l}\text { Mittelwert nach } \\
\text { nCPAP-Therapie } \pm \\
\text { Standardabweichung }\end{array}$ & Signifikanz \\
\hline Nach 1 Jahr (n=42) & & & \\
VCIN (\%Soll) & $93,27 \pm 13,45$ & $97,89 \pm 12,61$ & $\mathrm{p}<0,05$ \\
FEV $\%$ VC (\%) & $77,91 \pm 10,55$ & $76,29 \pm 10,56$ & $\mathrm{p}<0,05$ \\
Nach 2 Jahren (n=23) & & & \\
FEV $1 \%$ VC(\%) & $77,12 \pm 6,66$ & $72,98 \pm 9,90$ & $\mathrm{p}<0,05$ \\
FEV $1 \%$ VC (\% Soll) & $99,35 \pm 8,59$ & $94,70 \pm 12,60$ & $\mathrm{p}<0,05$ \\
Nach 3 Jahren (n=25) & & & \\
VCIN (\%Soll) & $88,60 \pm 13,14$ & $93,28 \pm 13,61$ & $\mathrm{p}<0,05$ \\
FEV $\%$ \%C (\%) & $79,02 \pm 9,82$ & $75,38 \pm 10,28$ & $\mathrm{p}<0,05$ \\
FEV $1 \%$ VC (\%Soll) & $100,94 \pm 11,95$ & $96,89 \pm 12,56$ & $\mathrm{p}<0,05$ \\
\hline
\end{tabular}

\section{Diskussion}

In der vorliegenden retrospektiven Studie wurden 228 Patienten mit einem erstdiagnostizierten OSAS untersucht. Bei allen Patienten erfolgte eine Einstellung auf eine nCPAP-Therapie und eine Verlaufsuntersuchung nach 1, 2 und/oder 3 Jahren. Sowohl bei der Erst- wie auch bei den jeweiligen Verlaufsuntersuchungen erfolgte eine polysomnographische Therapiekontrolle und eine Lungenfunktionsprüfung bestehend aus Blutgasanalyse, Spirometrie mit Fluss-Volumen-Kurve und Bodyplethysmographie mit dem Ziel, etwaige Veränderungen unter nCPAP-Therapie zu dokumentieren. Von der Teilnahme an der Untersuchung ausgeschlossen wurden Patienten, bei denen eine koprävalente obstruktive oder restriktive Lungenerkrankung bei der Erstuntersuchung vorlag oder die die nCPAP-Therapie nicht regelmäßig anwendeten (Anamnese, Betriebsstundenzähler). Unter den 228 Patienten befanden sich 69 (30,03\%) aktive Raucher, bei denen eine chronische Bronchitis im Sinne der GOLD-Klassifikation der COPD Stadium 0 (at Risk) angenommen werden muss. Alle Patienten wurden in Anlehnung an die Empfehlungen der Deutschen Gesellschaft für Schlafforschung und Schlafmedizin (DGSM) und der Deutschen Gesellschaft für Pneumologie (DGP) nach einer initialen Therapiekontrolle nach 3 Monaten in jährlichen Intervallen aufgefordert, an einer Kontrolluntersuchung ihrer nCPAP-Therapie teilzunehmen. In dieser Studie wurden die Ergebnisse der Verlaufsuntersuchung nach 1, 2 und 3 Jahren herangezogen. Da nicht alle Patienten zu allen Jahreskontrollen erschienen, wurden die bei den Kontrollen erhobenen Befunde mit den jeweiligen Ausgangsbefunden der Patienten verglichen, so dass die Kollektive nach 1, 2 und 3 Therapiejahren aus unterschiedlichen Teilkollektiven der erstuntersuchten Patienten zusammengesetzt waren. Von den Teilnehmern der Untersuchungen nach 2 und 3 Jahren waren jeweils etwa 50 Prozent schon zur Kontrolle nach 1 Therapiejahr erschienen. Bei diesen mehrfach nachuntersuchten Patienten ergaben sich jedoch keine von den lediglich einmalig nachuntersuchten Patienten abweichenden Befunde.

Alle Patienten zeigten anamnestisch sowie aufgrund der durch Betriebsstundenzähler kontrollierten nCPAP-Anwendung eine sehr gute Therapie-Compliance [11]. Somit ist einerseits sichergestellt, dass die nCPAP-Therapie ihren potenziellen Effekt auf die Lungenfunktion auswirken konnte. Andererseits ist zu diskutieren, dass Therapieabbrecher auch nicht zu den Nachuntersuchungen erschienen, wodurch die nachlassende Größe der Kontrollkollektive zu erklären ist. Im Beobachtungsintervall war eine signifikante Erhöhung des für eine effektive Therapie des OSAS erforderlichen nCPAP-Druckes notwendig, begründbar mit einer Verschlechterung des Krankheitsbildes (OSAS) oder einem besseren Nachtschlaf im Schlaflabor als Gewöhnungseffekt. Veränderungen des Körpergewichtes bzw. des BMI konnten als Ursache einer Verschlechterung des OSAS im Beobachtungsintervall ausgeschlossen werden [13].

\section{Einfluss der nCPAP-Therapie auf die Blutgaswerte}

Die Blutgasanalyse erfolgte kapillär aus dem hyperämisierten Ohrläppchen. Der Sauerstoffpartialdruck am Tage zeigte im Beobachtungsintervall keine signifikante Veränderung. Der Kohlendioxidpartialdruck war allerdings bei der dritten Kontrolluntersuchung gering, aber signifikant gesunken. Ein ähnliches Ergebnis erhielten Hein und Magnussen [7]. Auch bei ihnen fand sich nach $27 \pm 23$ Monaten im Gesamtkollektiv keine signifikante Veränderung der Partialdrücke. Vor Therapiebeginn hypoxämische Patienten profitierten aber von nCPAP, indem sich der Sauerstoffpartialdruck signifikant verbesserte. Chaouat u. Mitarb. [8] konnten nach fünf Therapiejahren ebenfalls keine signifikante Änderung des Sauerstoffpartialdruckes feststellen, während sie bei der Messung des Kohlendioxidpartialdruckes im Gegensatz zu uns eine leichte, aber doch signifikante Steigerung beobachteten. Wiederum andere Ergebnisse präsentierten Sforza u. Mitarb., welche nach einem nCPAP-Therapiejahr eine Verbesserung des Sauerstoffpartialdruckes fanden [9]. Der Kohlendioxidpartialdruck sank nicht im Gesamtkollektiv, sondern nur bei Patienten, die vor Therapiebeginn hyperkapnisch waren. Tun u. Mitarb. haben dagegen festgestellt, dass schon nach zwei Wochen nCPAP-Therapie der Sauerstoffpartialdruck signifikant stieg, während der des Kohlendioxids signifikant sank [10]. Kosaka u. Mitarb. fanden bei ihren Patienten nach 22 Monaten unter 
nCPAP-Therapie folgende Veränderungen der Blutgase: Der Sauerstoffpartialdruck stieg und der Kohlendioxidpartialdruck sank signifikant, während die Parameter der Lungenfunktionsanalyse gleich blieben [14]. Die Autoren hielten dies für einen Hinweis darauf, dass die nCPAP-Therapie den Gasaustausch verbessert. Ganz Ähnliches konnten Verbracken u. Mitarb. feststellen. Nach mindestens einem Monat nCPAP-Therapie hatte sich bei den Probanden mit normalen Lungenfunktionswerten der Gasaustausch signifikant verbessert [15]. In einer von Vazquez u. Mitarb. durchgeführten Untersuchung über die Wirkung von nCPAP auf den Gasaustausch an zwei Gruppen mit OSAS und zwar mit und ohne koprävalente chronisch obstruktive Lungenerkrankung zeigte sich bei den Patienten, die an beiden Erkrankungen litten, ein signifikanter Rückgang des $\mathrm{PaCO}_{2}$. Bei vorher hypoxämischen Personen stieg der Sauerstoffpartialdruck signifikant an. Diejenigen Patienten, welche nur an einem OSAS ohne Koprävalenz einer Lungenerkrankung litten, zeigten keine Veränderungen der Blutgase [16]. Unklar ist, ob die Atemantwort für $\mathrm{CO}_{2}$ durch die CPAP-Therapie verändert wird, hier ist die Datenlage in der Literatur widersprüchlich $[10,17,18]$. In Übereinstimmung mit den hier präsentierten Daten hat die nCPAP-Therapie zusammenfassend keinen bedeutenden Einfluss auf die Blutgasparameter am Tage $\left(\mathrm{PaO}_{2}, \mathrm{PaCO}_{2}\right)$ bei Lungengesunden, kann aber den Gasaustausch beziehungsweise die Ventilation bei entsprechend pulmonal erkrankten Patienten verbessern.

\section{Einfluss der nCPAP-Therapie auf die Lungenfunktion}

Im Beobachtungszeitraum von 3 nCPAP-Therapiejahren zeigte sich konstant eine signifikante Zunahme der VCIN, insbesondere bei den Rauchern. Als wahrscheinlichste Erklärung für die beobachtete Abnahme der MEF 50 und $\mathrm{FEV}_{1} \% \mathrm{VC}$ bei unveränderter $\mathrm{FEV}_{1}$ kann die Verbesserung der Vitalkapazität gelten, so dass sich MEF 50 und die relative Einsekundenkapazität einfach rechnerisch verändert haben. Da die Veränderungen der VCIN und diejenigen der anderen Parameter jedoch nicht immer zeitlich parallel festzustellen sind, können andere Ursachen für die signifikanten Veränderungen der unterschiedlichen Parameter nicht völlig ausgeschlossen werden. Insbesondere die signifikante Abnahme der MEF-50-Werte im Verlauf kann als Hinweis für einen beginnenden Elastizitätsverlust der Lunge oder als Zeichen einer unspezifischen bronchialen Hyperreaktivität gewertet werden [19]. Eine entsprechende Testung der bronchialen Reagibilität wurde jedoch im Rahmen dieser Untersuchung nicht durchgeführt. Bei der Analyse der Ausgangswerte fand sich bei den Rauchern eine mittlere VCIN von $90 \%$ des Sollwertes bei gleichzeitig erhöhtem Residualvolumen (RV), was im Sinne einer leichtgradigen atemwegsobstruktionsbedingten Lungenüberblähung zu werten ist [19]. Diese mögliche Obstruktion, für welche auch der erniedrigte mittlere MEF-50-Wert in der Ausgangsuntersuchung spräche, könnte durch die nCPAP-Therapie vermindert worden sein. Es ist möglich, dass insbesondere Raucher mit einer chronischen Bronchitis durch die nCPAP-Therapie eine Aufhebung von obstruktionsbedingten Ventilations-Inhomogenitäten erfahren, die sich in der Erhöhung der Vitalkapazität im Verlauf widerspiegelt [20]. Hardy und Anderson beschrieben bei mit positivem Druck beatmeten, an Muskeldystrophien leidenden Kindern eine Verbesserung der Vitalkapazität und eine verbesserte Möglichkeit des Abhustens von Sekret [21]. Ebenso fanden Tsai und Tsai bei ihren Patienten mit einem hyperreagiblem Bronchialsystem und vermehrter Schleimsekretion eine Symptomver- besserung durch positiven exspiratorischen Druck [22]. Ähnliche Mechanismen könnten bei den OSAS-Patienten vorliegen und so zu einer Steigerung der Vitalkapazität insbesondere bei den Rauchern führen.

Als weitere mögliche Erklärung für die Verbesserung der VCIN ist ein Übungseffekt bei der Durchführung der Lungenfunktionsuntersuchung zu diskutieren. Gerade das Untersuchungsmanöver, dass zur Ermittlung der VCIN notwendig ist, ist für den Patienten schwierig durchzuführen. Je öfter eine Person dieses Manöver durchführt, desto leichter wird es ihr fallen, ein optimales Ergebnis zu erzielen [19].

Vazquez u. Mitarb. konnten bei Patienten mit einem OSAS ohne koprävalente Lungenerkrankung unter nCPAP-Therapie keine signifikanten Veränderungen in der Lungenfunktion im Beobachtungsintervall von 12 Monaten feststellen [16]. Ebenso konnten Kosaka u. Mitarb. bei 25 Patienten mit OSAS nach 22 Monaten nCPAP-Therapie keine signifikanten Veränderungen in der Spirometrie beobachten [14]. Auch Hein und Magnussen konnten lungenfunktionsanalytisch nach durchschnittlich 27 Monaten keine Auswirkungen der nCPAP-Therapie auf die Atemmechanik nachweisen [7].

Demgegenüber zeigten Chaouat u. Mitarb. in ihrem Patientenkollektiv, welches allerdings einen hohen Raucheranteil von 77\% hatte, eine signifikante Verschlechterung der Einsekundenkapazität $\left(\mathrm{FEV}_{1}\right)$ nach einer nCPAP-Therapiezeit von ca. fünf Jahren [8]. Die von dieser Arbeitsgruppe beobachtete Verschlechterung der Einsekundenkapazität bei Rauchern findet durch unsere Untersuchung keine Bestätigung, wenngleich in unserem Kollektiv ebenfalls eine Verschlechterung von $\mathrm{FEV}_{1} \% \mathrm{VC}$ nachgewiesen wurde, was allerdings auf die Verbesserung von VCIN zurückzuführen ist.

Nach den Ergebnissen einer Longitudinalbeobachtung über 6 respektive 18 Monate profitierten Patienten mit einem OSAS und einer koprävalenten chronisch obstruktiven Atemwegserkrankung von nCPAP mit einer Verbesserung des Gasaustausches sowie einer Zunahme von $\mathrm{FEV}_{1}$ und der forcierten Vitalkapazität, korrespondierend zu den Ergebnissen unserer Studie [23].

Eine Zunahme der totalen Lungenkapazität sowie des Residualvolumens im Sinne der Überblähung des Patienten durch den applizierten Überdruck ist denkbar [5], entsprechende Veränderungen der totalen Lungenkapazität und des prozentualen Residualvolumens waren jedoch in dieser Untersuchung nur einmalig in dem nach 2 Jahren nCPAP-Therapie nachuntersuchten Kollektiv nachweisbar gewesen. Da hier eine Übereinstimmung mit den Verlaufuntersuchungen nach 1 und 3 Jahren fehlten, ist am ehesten von einem kollektivspezifischen Faktor auszugehen, nicht aber eine systematische Nebenwirkung der nCPAP-Therapie anzunehmen. Zusammenfassend ist eine negative Auswirkung der nCPAP-Therapie auf die Lungenfunktion nicht belegt, Abweichung hiervon sind im Einzelfall jedoch sowohl im Sinne einer Verbesserung als auch einer Verschlechterung der Lungenfunktion möglich. 


\section{Schlussfolgerung}

Die Entwicklung einer obstruktiven oder restriktiven Ventilationsstörung wurde unter nCPAP-Therapie im Beobachtungsintervall von bis zu 3 Jahren nicht beobachtet. In der vorliegenden retrospektiven Untersuchung wurde unter nCPAP-Therapie eine Zunahme der Vitalkapazität gezeigt, die am ehesten durch einen Übungseffekt bei der Durchführung des Untersuchungsmanövers und durch Rekrutierung minderbelüfteter Lungenareale erklärbar ist. Verschlechterungen der MEF-50-Werte sowie des Tiffeneau-Wertes bei unveränderter Einsekundenkapazität sind durch die Relativität zur Verbesserung der Vitalkapazität erklärbar. Somit konnte ein eindeutiger negativer Einfluss der nCPAP-Therapie auf die Lungenfunktion im Verlauf von 3 Jahren in dem untersuchten Patientenkollektiv nicht gezeigt werden.

\section{Literatur}

${ }^{1}$ Sullivan CE, Issa FG, Berthon-Jones M et al. Reversal of obstructive sleep apnoea by continuous positive airway pressure applied through the nares. Lancet 1981; 1: 862-865

${ }^{2}$ American Sleep Disorders Association. Practice parameters for the treatment of snoring and obstructive sleep apnea. Sleep 1995; 18: $511-513$

${ }^{3}$ Hein H, Raschke F, Köhler D et al. Leitlinie zur Diagnostik und Therapie schlafbezogener Atmungsstörungen beim Erwachsenen. Pneumologie 2001; 53: 339-342

${ }^{4}$ White J, Cates C, Wright J. Continuous positive airways pressure for obstructive sleep apnoea. Cochrane Database Syst Rev 2002; (2): CD001106

${ }^{5}$ O'Donoghue FJ, Catcheside PG, Jordan AS et al. Effect of CPAP on intrinsic PEEP, inspiratory effort, and lung volume in severe stable COPD. Thorax 2002; 57: $533-539$

${ }^{6}$ Heulitt MJ, Holt SJ, Wilson S et al. Effects of continuous positive airway pressure/positive endexpiratory pressure and pressure-support ventilation on work of breathing, using an animal model. Respir Care 2003. 48: 689-696

${ }^{7}$ Hein H, Magnussen H. Die nCPAP-Therapie beeinflusst nicht die Atemmechanik von Patienten mit schlafbezogenen Atmungsstörungen. Pneumologie 2000; 54: 335 - 337

${ }^{8}$ Chaouat A, Weitzenblum E, Kessler R et al. Five-year effects of nasal continuous positive airway pressure in obstructive sleep apnoea syndrome. Eur Respir J 1997; 10: 2578-2582
${ }^{9}$ Sforza E, Krieger J, Weitzenblum E et al. Long-term effects of treatment with nasal continuous airway pressure on daytime lung function and pulmonary hemodynamics in patiets with obstructive sleep apnea. Am Rev Resp Dis 1990; 141: 866-870

${ }^{10}$ Tun Y, Hida W, Okabe S et al. Effects of nasal continuous positive airway pressure on awake ventilatory responses to hypoxia and hypocapnia in patients with obstructive sleep apnea. Tohoku J Exp Med 2000; 190: $157-168$

${ }^{11}$ Kribbs N, Pack A, Klein L et al. Objective Measurement of patterns of nasal CPAP use by patients with obstructive sleep apnea. Am Rev Respir Dis 1993; 147: 887-895

12 Quanjer PhH, Europäische Gemeinschaft für Kohle und Stahl (EGKS). Standardized Lung Function Testing Bull Europ Physiopath Resp 1983/1993; 19 (suppl 5)/6 (suppl 16): $11-21$

${ }^{13}$ Geisler P. Veränderungen des nCPAP-Druckes bei wiederholten Kontrollen bei Patienten mit obstruktiver Schlafapnoe. Wiener Med Wochenschrift 1995; 145: 506-507

${ }^{14}$ Kosaka N, Akashiba T, Minemura $\mathrm{H}$ et al. Long-term effects of nasal continuous airway pressure on pulmonary function and blood gases data in patients with obstructive sleep apnea syndrome. Nihon Kokyuki Gakkai Zasshi 1998; 36: 1011 - 1016

${ }^{15}$ Verbracken J, Willemen M, De Cock W et al. Continuous positive airways pressure and lung inflation in sleep apnea patients. Respiration 2001; 68: $357-364$

${ }^{16}$ Vazquez Oliva R, Cano Gomez S, Capote Gil F et al. Effects of nasal continuous positive airway pressure on lung function in patients with sleep obstructive apnea syndrome. Archivos de Broncopneumologia 1995; 31: $18-22$

${ }^{17}$ Verbraecken J, Willemen M, De Cock W et al. Influence of longterm CPAP therapy on $\mathrm{CO}_{2}$ ) drive in patients with obstructive sleep apnea. Respir Physiol 2000; 123: 121 - 130

${ }^{18}$ Moura SM, Bittencourt LR, Bagnato MC et al. Acute effect of nasal continuous positive air pressure on the ventilatory control of patients with obstructive sleep apnea. Respiration 2001; 68: 243-249

${ }^{19}$ Baur X. Lungenfunktionsprüfung und Allergiediagnostik. München: Dustri-Verlag, 1998

20 Tusman G, Bohm SH, Tempra A et al. Effects of recruitment maneuver on atelectasis in anesthesized children. Anesthesiology 2003; 98: $14-22$

${ }^{21}$ Hardy KA, Anderson BD. Noninvasive clearance of airway secretions. Respir Care Clin N Am 1996; 2: 323 - 345

22 Tsai CF, Tsai JJ. Effectiveness of a positive expiratory pressure device in conjunction with $\beta_{2}$-agonist nebulization therapy for brochial asthma. J Microbiol Immunol Infect 2001; 34: 92 -96

${ }^{23}$ De Miguel J, Sanchez-Alarcos JM, Alvarez-Sala R et al. Long-term effects of treatment with nasal continuous positive airway pressure on lung function in patients with overlap syndrome. Sleep Breath 2002; 6: $3-10$ 